Honam Mathematical J. 36 (2014), No. 1, pp. 141-146

http://dx.doi.org/10.5831/HMJ.2014.36.1.141

\title{
VARIATIONS OF THE LENGTH INTEGRAL
}

\author{
Yong-Soo Pyo*, Won Tae Oh, and Heui-Sang Son
}

\begin{abstract}
In this paper, we obtain a necessary and sufficient condition for the second variation of an arbitrarily given smooth variation of a geodesic on a Riemannian manifold to be 0 .
\end{abstract}

\section{Introduction}

Let $\tau_{s}:[0,1] \rightarrow M(-\varepsilon<s<\varepsilon)$ be a smooth variation of a geodesic $\tau_{0}=\tau=x_{t}(0 \leq t \leq 1)$ on a Riemannian manifold $(M, g)$ such that $\tau_{s}(0)=x_{0}$ and $\tau_{s}(1)=x_{1}$ for every $s \in(-\varepsilon, \varepsilon)$. We put

$$
L(s):=L\left(\tau_{s}\right):=\int_{0}^{1}\left\|\frac{d \tau_{s}}{d t}\right\| d t
$$

and calculate the second variation $\left(d^{2} L(s) / d s^{2}\right)_{s=0}$. The second variation is expressed in terms of the sectional curvature $([1,2,3,4])$ and the variation vector field along the curve $\tau=x_{t}(0 \leq t \leq 1)$. And then, we get a necessary and sufficient condition for the second variation $\left(d^{2} L(s) / d s^{2}\right)_{s=0}$ to be 0 .

These calculus methods in variation problems are very useful in the study on natural sciences. But, in most of references, the calculus of variations is insufficient and omitted. In this paper, we make a minute and detailed poof of the calculus parts which are insufficient and omitted in variation problems of the length integral.

Received December 22, 2013. Accepted January 27, 2014.

2010 Mathematics Subject Classification. 53C30, 53C25.

Key words and phrases. the first (second) variation, variational vector field, geodesic.

${ }^{*}$ Corresponding author 


\section{The first and second variations of the length integral}

Let $(M, g)$ be a complete Riemannian manifold and $\nabla$ the Levi-Civita connection for the Riemannian metric $g$. For a $C^{\infty}$-curve $\tau=x_{t}(0 \leq$ $t \leq 1)$ on $M$, let

$$
\phi:(t, s) \in[0,1] \times(-\varepsilon, \varepsilon) \mapsto \phi(t, s) \in M
$$

be a $C^{\infty}$-map which satisfies

$$
\phi(t, 0)=x_{t} .
$$

Such a map $\phi$ is called a variation of $\tau=x_{t}(0 \leq t \leq 1)$. From now on, we assume that $\tau=x_{t}(0 \leq t \leq 1)$ is parametrized by its arc length. Let $\tau_{s}:[0,1] \rightarrow M(-\varepsilon<s<\varepsilon)$ be a $C^{\infty}$-map which is defined by $\tau_{s}(t):=\phi(t, s)$. The length $L(s)$ of $\tau_{s}$ is given by

$$
L(s):=L\left(\tau_{s}\right)=\int_{0}^{1} \sqrt{g\left(\phi_{*}(\partial / \partial t)_{(t, s)}, \phi_{*}(\partial / \partial t)_{(t, s)}\right)} d t .
$$

Let $\phi_{*}(\partial / \partial t)_{(t, s)}$ and $\phi_{*}(\partial / \partial s)_{(t, s)}$ are defined on $[0,1] \times(-\varepsilon, \varepsilon)$. Moreover, we define a vector field $X_{t}$ along the curve $\tau=x_{t}(0 \leq t \leq 1)$ by

$$
X_{t}:=\phi_{*}(\partial / \partial s)_{(t, 0)} \quad(0 \leq t \leq 1) .
$$

Such a vector field $X_{t}(0 \leq t \leq 1)$ along the curve $\tau=x_{t}(0 \leq t \leq 1)$ is called the variation vector field along $\phi$. In this paper, we assume that $\phi(0, s)=x_{0}$ and $\phi(1, s)=x_{1}, s \in(-\varepsilon, \varepsilon)$, for any variation $\phi$ of the curve $\tau=x_{t}(0 \leq t \leq 1)$. Then we have

$$
X_{0}=0 \in T_{x_{0}}(M), \quad X_{1}=0 \in T_{x_{1}}(M) .
$$

Now we calculate the first variation $(d L / d s)_{s=0}$ of $L(s)$. First of all, we get

$$
\begin{array}{r}
\frac{\partial}{\partial s}\left(\sqrt{g\left(\phi_{*}(\partial / \partial t)_{(t, s)}, \phi_{*}(\partial / \partial t)_{(t, s)}\right)}\right) \\
=\frac{g\left(\nabla_{s} \phi_{*}(\partial / \partial t)_{(t, s)}, \phi_{*}(\partial / \partial t)_{(t, s)}\right)}{\sqrt{g\left(\phi_{*}(\partial / \partial t)_{(t, s)}, \phi_{*}(\partial / \partial t)_{(t, s)}\right)}} .
\end{array}
$$

Since

$$
\begin{aligned}
\nabla_{s} \phi_{*}(\partial / \partial t)_{(t, s)}- & \nabla_{t} \phi_{*}(\partial / \partial s)_{(t, s)}-\phi_{*}\left[(\partial / \partial s)_{(t, s)},(\partial / \partial t)_{(t, s)}\right] \\
& =T^{\nabla}\left(\phi_{*}(\partial / \partial s)_{(t, s)}, \phi_{*}(\partial / \partial t)_{(t, s)}\right)
\end{aligned}
$$

and $T^{\nabla}=0$ (cf, [3, Lemma 1.16, p. 151]), we obtain on $[0,1] \times(-\varepsilon, \varepsilon)$

$$
\nabla_{s} \phi_{*}(\partial / \partial t)_{(t, s)}=\nabla_{t} \phi_{*}(\partial / \partial s)_{(t, s)} .
$$


Here, $T^{\nabla}$ is the torsion of $\nabla$. We get from (2.2) and (2.5)

$$
\phi_{*}(\partial / \partial t)_{(t, 0)}=x_{t}^{\prime}, \quad\left(\nabla_{s} \phi_{*}(\partial / \partial t)_{(t, s)}\right)_{s=0}=\nabla_{t} X_{t} .
$$

From (2.1), (2.4) and (2.6), we obtain

$$
\left(\frac{d L(s)}{d s}\right)_{s=0}=\int_{0}^{1} g\left(\nabla_{t} X_{t}, x_{t}^{\prime}\right) d t .
$$

Moreover, we get

$$
g\left(\nabla_{t} X_{t}, x_{t}^{\prime}\right)=\frac{d}{d t}\left(g\left(X_{t}, x_{t}^{\prime}\right)\right)-g\left(X_{t}, \nabla_{t} x_{t}^{\prime}\right) .
$$

By the help of (2.7) and (2.8), we have

$$
\begin{aligned}
\left(\frac{d L(s)}{d s}\right)_{s=0} & =\int_{0}^{1}\left\{\frac{d}{d t}\left(g\left(X_{t}, x_{t}^{\prime}\right)\right)-g\left(X_{t}, \nabla_{t} x_{t}^{\prime}\right)\right\} d t \\
& =\left[g\left(X_{t}, x_{t}^{\prime}\right)\right]_{0}^{1}-\int_{0}^{1} g\left(X_{t}, \nabla_{t} x_{t}^{\prime}\right) d t .
\end{aligned}
$$

Since $X_{0}=0 \in T_{x_{0}}(M)$ and $X_{1}=0 \in T_{x_{1}}(M)$ from (2.3), we get from $(2.9)$

$$
\left(\frac{d L(s)}{d s}\right)_{s=0}=-\int_{0}^{1} g\left(X_{t}, \nabla_{t} x_{t}^{\prime}\right) d t
$$

Hence we have the following theorem.

Theorem 2.1. Let $\tau_{s}:[0,1] \rightarrow M(-\varepsilon<s<\varepsilon)$ be an arbitrarily given smooth variation of $\tau=x_{t}(0 \leq t \leq 1)$ such that $\tau_{s}(0)=x_{0}$ and $\tau_{s}(1)=x_{1}$ for every $s \in(-\varepsilon, \varepsilon)$. Then, $(d L(s) / d s)_{s=0}=0$ if and only if $\nabla_{t} x_{t}^{\prime}=0$ for every $t \in(0,1)$, that is, $\tau=x_{t}(0 \leq t \leq 1)$ is a geodesic in the Riemannian manifold $(M, g)$.

Next, we calculate the second variation $\left(d^{2} L(s) / d s^{2}\right)_{s=0}$ of the geodesic $\tau=x_{t}(0 \leq t \leq 1)$. From (2.4) and (2.5), we get

$$
\begin{aligned}
\frac{\partial^{2}}{\partial s^{2}}\left(\sqrt{g\left(\phi_{*}(\partial / \partial t)_{(t, s)}, \phi_{*}(\partial / \partial t)_{(t, s)}\right)}\right) & g\left(\nabla_{s} \nabla_{t} \phi_{*}(\partial / \partial s)_{(t, s)}, \phi_{*}(\partial / \partial t)_{(t, s)}\right)+\left\|\nabla_{t} \phi_{*}(\partial / \partial s)_{(t, s)}\right\|^{2} \\
= & \left\{g\left(\phi_{*}(\partial / \partial t)_{(t, s)}, \phi_{*}(\partial / \partial t)_{(t, s)}\right)\right\}^{\frac{1}{2}} \\
& -\frac{\left\{g\left(\nabla_{t} \phi_{*}(\partial / \partial s)_{(t, s)}, \phi_{*}(\partial / \partial t)_{(t, s)}\right)\right\}^{2}}{\left\{g\left(\phi_{*}(\partial / \partial t)_{(t, s)}, \phi_{*}(\partial / \partial t)_{(t, s)}\right)\right\}^{\frac{3}{2}}} .
\end{aligned}
$$


Moreover, the following is well known ([2, Proposition 10, p. 261]):

$$
\begin{aligned}
& \nabla_{s} \nabla_{t} \phi_{*}(\partial / \partial s)_{(t, s)}-\nabla_{t} \nabla_{s} \phi_{*}(\partial / \partial s)_{(t, s)} \\
& \quad=R\left(\phi_{*}(\partial / \partial s)_{(t, s)}, \phi_{*}(\partial / \partial t)_{(t, s)}\right) \phi_{*}(\partial / \partial s)_{(t, s)} .
\end{aligned}
$$

Here, $R$ is the curvature tensor field on $(M, g)$. Furthermore,

$$
\begin{aligned}
g\left(\nabla_{t} \nabla_{s} \phi_{*}(\partial / \partial s)_{(t, s)}, \phi_{*}(\partial / \partial t)_{(t, s)}\right) & \\
= & \frac{d}{d t}\left\{g\left(\nabla_{s} \phi_{*}(\partial / \partial s)_{(t, s)}, \phi_{*}(\partial / \partial t)_{(t, s)}\right)\right\} \\
& -g\left(\nabla_{s} \phi_{*}(\partial / \partial s)_{(t, s)}, \nabla_{t} \phi_{*}(\partial / \partial t)_{(t, s)}\right) .
\end{aligned}
$$

By the help of (2.6), (2.10), (2.11) and (2.12), we obtain

$$
\begin{aligned}
\left(\frac{d^{2} L(s)}{d s^{2}}\right)_{s=0}= & {\left[\left(g\left(\nabla_{s} \phi_{*}(\partial / \partial s)_{(t, s)}, \phi_{*}(\partial / \partial t)_{(t, s)}\right)\right)_{s=0}\right]_{t=0}^{1} } \\
& +\int_{0}^{1}\left\{g\left(R\left(X_{t}, x_{t}^{\prime}\right) X_{t}, x_{t}^{\prime}\right)+\left\|\nabla_{t} X_{t}\right\|^{2}\right. \\
& \left.-\left(g\left(\nabla_{t} X_{t}, x_{t}^{\prime}\right)\right)^{2}\right\} d t .
\end{aligned}
$$

Let $X_{t}^{\perp}(0 \leq t \leq 1)$ be the component of $X_{t}$ perpendicular to the geodesic $\tau=x_{t}(0 \leq t \leq 1)$, that is,

$$
X_{t}^{\perp}=X_{t}-g\left(X_{t}, x_{t}^{\prime}\right) x_{t}^{\prime} .
$$

Since $\tau_{s}(0)=\phi(0, s)=x_{0}$ and $\tau_{s}(1)=\phi(1, s)=x_{1}$ for every $s \in(-\varepsilon, \varepsilon)$, we get

$$
\nabla_{s} \phi_{*}(\partial / \partial s)_{(0, s)}=0, \quad \nabla_{s} \phi_{*}(\partial / \partial s)_{(1, s)}=0 .
$$

From (2.14), we obtain

$$
\begin{aligned}
& \nabla_{t} X_{t}=\nabla_{t} X_{t}^{\perp}+\frac{d\left(g\left(X_{t}, x_{t}^{\prime}\right)\right)}{d t} x_{t}^{\prime}, \\
& 0=\frac{d\left(g\left(X_{t}^{\perp}, x_{t}^{\prime}\right)\right)}{d t}=g\left(\nabla_{t} X_{t}^{\perp}, x_{t}^{\prime}\right),
\end{aligned}
$$

because $x_{t}(0 \leq t \leq 1)$ is a geodesic in the Riemannian manifold $(M, g)$. From (2.16), we get

$$
\left\|\nabla_{t} X_{t}\right\|^{2}-\left(g\left(\nabla_{t} X_{t}, x_{t}^{\prime}\right)\right)^{2}=\left\|\nabla_{t} X_{t}^{\perp}\right\|^{2} .
$$

We obtain from (2.13), (2.14), (2.15) and (2.17)

$$
\left(\frac{d^{2} L(s)}{d s^{2}}\right)_{s=0}=\int_{0}^{1}\left\{\left\|\nabla_{t} X_{t}^{\perp}\right\|^{2}-g\left(R\left(X_{t}^{\perp}, x_{t}^{\prime}\right) x_{t}^{\prime}, X_{t}^{\perp}\right)\right\} d t .
$$

Thus, we get the following theorem. 
Theorem 2.2. Let $\tau_{s}:[0,1] \rightarrow M(-\varepsilon<s<\varepsilon)$ be an arbitrarily given variation of a geodesic $\tau_{0}=\tau=x_{t}(0 \leq t \leq 1)$ on $(M, g)$ such that $\tau_{s}(0)=x_{0}$ and $\tau_{s}(1)=x_{1}$ for every $s \in(-\varepsilon, \varepsilon)$. Then the second variation $\left(d^{2} L / d s^{2}\right)_{s=0}$ is given as follows:

$$
\begin{aligned}
\left(\frac{d^{2} L(s)}{d s^{2}}\right)_{s=0} & =\int_{0}^{1}\left\{\left\|\nabla_{t} X_{t}^{\perp}\right\|^{2}-g\left(R\left(X_{t}^{\perp}, x_{t}^{\prime}\right) x_{t}^{\prime}, X_{t}^{\perp}\right)\right\} d t \\
& =\int_{0}^{1}\left\{\left\|\nabla_{t} X_{t}^{\perp}\right\|^{2}-\left\|X_{t}^{\perp}\right\|^{2} \sigma\left(X_{t}^{\perp}, x_{t}^{\prime}\right)\right\} d t
\end{aligned}
$$

where $\sigma\left(X_{t}^{\perp}, x_{t}^{\prime}\right)$ is the sectional curvature determined by $\left\{X_{t}^{\perp}, x_{t}^{\prime}\right\}$.

By virtue of Theorem 2.2, we obtain the following corollary.

Corollary 2.3. Assume that $(M, g)$ is a space of constant negative. For an arbitrarily given variation $\tau_{s}:[0,1] \rightarrow M(-\varepsilon<s<\varepsilon)$ of a geodesic $\tau_{0}=\tau=x_{t}(0 \leq t \leq 1)$ on $(M, g)$ such that $\tau_{s}(0)=x_{0}$ and $\tau_{s}(1)=x_{1}$ for every $s \in(-\varepsilon, \varepsilon)$, a necessary and sufficient condition for the second variation $\left(d^{2} L / d s^{2}\right)_{s=0}$ to be 0 is $X_{t}^{\perp}=0(0 \leq t \leq 1)$.

\section{References}

[1] J.-S. Park, Curvature on $S U(3) / T(k, l)$, Kyushu J. Math. 67(1) (2013), 55-65.

[2] Y.-S. Pyo, H.-J. Shin and J.-S. Park, Scalar curvatures on $S U(3) / T(k, l)$, Honam Math. J. 33(4) (2011), 547-556.

[3] M. Spivak, A Comprehensive Introduction to Differential Geometry II, Publish or Perish, Inc. Berkeley, 1979.

[4] H. Urakawa, Calculus of Variations and Harmonic Maps (in Japanese), Shokabo Publ., 1990.

Yong-Soo Pyo

Department of Applied Mathematics, Pukyong National University,

Busan 608-737, Korea.

E-mail : yspyo@pknu.ac.kr

Won Tae Oh

Department of Mathematics, Chungbuk National University, Cheongju 361-763, Korea.

E-mail : wntoh@cbnu.ac.kr 
Heui-Sang Son

Department of Applied Mathematics, Pukyong National University, Busan 608-737, Korea.

E-mail : sonheuisang@hanmail.net 